\title{
Let's talk about thal: How communication can improve quality of life
}

\section{Laurice M. Levine}

\author{
Thalassemia Patient, Thalassemia Program Consultant, Children's Hospital Los Angeles, \\ Kingston, WA USA
}

In many parts of the world, research, improved technology, and better medicine have enabled people with thalassemia to live longer. It is tragic that due to global disparity in healthcare, in areas of the world where thalassemia is most prevalent, the mortality rates are high, and often, patients do not survive past adolescence.

Each stage of life holds different challenges for people with thalassemia, and if patients are fortunate enough to reach adulthood, then they are faced with a new set of challenges uncommon to pediatric patients. Providers who have dedicated their careers to improving care now must work toward helping patients achieve a high quality of adult life by addressing such struggles. However, there is one topic that affects patients and providers universally at every stage of life-implementation of the concept can be easy, and it is free: that is COMMUNICATION!

I am a 45-year-old thalassemia patient. I have 17 years of experience working in thalassemia outreach and advocacy at a children's hospital, I am currently a thalassemia program consultant at Children's Hospital Los Angeles, and I follow topics related to thalassemia on over 60 social media sites. Consequently, I have become more aware of patient needs, challenges, and obstacles on a global level. My thought process for this presentation led to a goal of discussing quality of life in a way that considered patients all over the world, not just the United States. The common factor that can improve quality of life for patients around the globe is a high level of communication.

Communication can begin upon diagnosis. Though communication is vital to surviving with thalassemia and a key to quality of life, it is a challenge for many people, and at times, a curse. People do not want to talk about thalassemia, and often, they go into hiding. What does that achieve?

In this presentation, I will explain the model of communication that I developed, including the facets of communication, why it is vital, and how many of the burdens of thalassemia can be overcome through communication, with an emphasis on some adult issues: disclosure, relationships, education/employment, secondary disease, and insurance.

To achieve successful communication, barriers and considerations need to be recognized.

Barriers to communication:

- Thalassemia affects many various ethnicities-language barriers.

Correspondence: Laurice M. Levine, 65 Orange Ave. Larkspur, California 94939 USA. Tel.: 360-860-2023.

E-mail: LauriceLevine@gmail.com

This work is licensed under a Creative Commons Attribution 4.0 License (by-nc 4.0).

CC Copyright L.M. Levine, 2018

Licensee PAGEPress, Italy

Thalassemia Reports 2018; 8:7490

doi:10.4081/thal.2018.7490
- There can be a lack of understanding of medical information.

- Thalassemia is complicated and difficult to explain.

- Fear of rejection; cultural and social stigmas attached to having a chronic illness, especially a blood disorder.

- Technology-look up from the phone!

Considerations in methods of communication:

- Age: upon diagnosis, the parent communicates for the child; throughout life, the patient becomes more autonomous.

- Culture: body language and eye contact. Out of respect for providers, patients may not ask questions.

Model for communication includes:

- Communication with oneself.

Self-worth/self-esteem.

- "I" messages: I am worthy, I am lovable, etc.

- I am a person with thalassemia, not a thalassemic.

- There is no NORMAL. All people on the planet have differences in their lives and health. Where diversity lives, normal does not exist.

- Communication with family.

- Tell family members to get tested for trait.

- Siblings-differences in their health and feelings around the disease.

- For the health and well-being of all.

- Communication with peers in the thalassemia community.

- Support each other.

- Learn from each other.

- Social media can facilitate discussion, support, and information sharing.

- Help/advise/listen.

- Don't get caught up in politics.

- Communication with friends.

- Socialization.

- Disclosure-to tell or not to tell.

- Intimate relationships-at what point in a relationship do you disclose?

- Communication with the community.

- School.

○ Absence.

- Participation.

$\circ$ Educate peers and teachers.

- Work.

$\circ$ Absence.

$\circ$ Work ethic-the need to prove yourself.

- Communication with and among providers.

- Patient-provider.

- Standards of care.

- International standards.

- Modification by country. The standard is specific to each country-care depends on education, infrastructure, availability of medical supplies and medication.

- Collaboration; develop a partnership.

$\circ$ New treatments. 
○ Be informed.

○ Ask questions.

- Provider-provider.

- Standards of care-agreement.

o Collaboration.

- Information on latest research.

- Transition (specific to location).

- Global communication.

- Organizations.

○ Thalassemia International Federation (TIF): www.thalassaemia.org.cy.

- Cooley's Anemia Foundation (CAF): www.thalassemia.org.

- Thalassemia Support Foundation (TSF): www. helpthals.org.

$\circ$ National Institutes of Health (NIH): www. nih.gov/.

$\circ$ Food and Drug Administration (FDA): www. fda.gov/.

○ European Medicines Agency (EMA): tinyurl.com/y7kbzn6q.

- Clinical trials.

○ www.thalassemia.org/learn-about-thalassemia/clinical-trials/.

○ www.clinicaltrials.gov/, then type in "thalassemia."

- Governments.

$\circ$ Laws affecting thalassemia community.

$\circ$ Insurance.

- Social media.

- Facebook, Twitter, Instagram.

○ Laurice M. Levine-thalassemia updates on Facebook.

- Advocacy.

- Provide community outreach-give talks on thalassemia; patient perspective. Attend health fairs, festivals.

- Awareness of thalassemia is vital. The more people know about thalassemia, the more likely it is that services will be provided to the community.

- Knowledge is power. If people learn about thalassemia, they will care about thalassemia.

- Change policy that affects people with rare diseases.

- Improved care. Work toward a cure.

\section{Conclusions}

Communication is the key to achieving a high quality of life. It is the foundation for care and can help toward a cure. Providers will learn the benefits of communication; they will have a clearer picture of what patients face. They can compassionately help patients achieve a higher quality of life by overcoming these challenges through using their voices.

Eradicate the word NORMAL. There are some challenges that are inherent to thalassemia regardless of what country the patient lives in: blood safety; access to care (even in the United States, many patients live far from centers of excellence); expertise; cost of care; social stigma and barriers that patients are working so hard to overcome. A primary way to overcome these challenges is to use our voices.

Strength does not come from physical capacity. It comes from an indomitable will.

Mahatma Gandhi

You must be the change you want to see in the world.

Mahatma Gandhi

References

Hossain et al. Orphanet Journal of Rare Diseases (2017) 12:93. 